\title{
Research on the Monitoring Technology of Multiple Elevators Based on Internet of Things and Industrial Configuration
}

\author{
Xueyan $\mathrm{Li}^{1, \mathrm{a}}$ \\ ${ }^{1}$ City College, Wuhan University of Science and Technology, Wuhan, 430083, China
}

Keywords: Internet of things, Industrial Configuration, Elevator monitoring

\begin{abstract}
To improve the reliability and safety of elevator operation we construct the Multiple Elevators monitoring system based on the Internet of Things and industrial configuration. It can reduce the waiting time when the elevator goes out of order. This paper introduces the concept of IoT technology and industrial configuration technology and designs the elevator monitoring system based on these two technologies and finally realizes the functions of problem alarming, reasons tracing and experiences sharing, of the monitoring system of multi elevators.
\end{abstract}

\section{Introduction of the Technology of IoT}

The Internet of Things is not only an important part of the new generation of information technology, but also an important stage of development of the information age. Internet of Things is connected to and based on the internet. It has two meanings. First, the core and foundation of things is still the Internet, is the extension and expansion of Internet based on the network; second, the extension and expansion of the client to any goods and articles, information exchange and communication, it is a matter of interest. The Internet of Things through intelligent perception, recognition technology and pervasive computing and communication technology is widely used in sensing, fusion network, and has therefore been called following the computer and the Internet information industry development of the world's third wave. Internet of Things is the development of Internet applications, rather than the Internet of Things is the network, rather than the Internet of Things is the business and application. Therefore, the application of innovation is the core of the development of the Internet of things, to the user experience as the core of innovation 2 is the soul of the development of the Internet of things. The Internet of Things is a huge network which is formed by the combination of the Internet and the Internet through various information sensing devices, and the real-time acquisition of any information which needs to be monitored, connected and interactive. The purpose is to realize the connection of objects and objects, objects and people, all the goods and the network to facilitate the identification, management and control. As the third wave of the development of information technology in the world, after the computer and the Internet, the Internet of Things has become an important part of the national science and technology development strategy. As special equipment industry practitioners, the Internet of Things technology applied to special equipment monitoring, maintenance and other fields, we need to think and need to be put into action as soon as possible.

\section{Basic Theory of Industrial Configuration}

The configuration of configuration software tools using the resources of computer and software, configuration software for monitoring and data acquisition software platform has the characteristics of project settings rich, flexible use, powerful, open interface, real-time and reliable operation etc.. The configuration software can monitor and control the automation process and equipment. It can collect all kinds of information from the automation process and equipment, and information to a graphic display more easy to understand way, the important information is transmitted by various means to the relevant personnel to perform the necessary analysis, information processing and storage, sends out the control command. Configuration data can only be identified by its manufacturing tools or other special tools. But the difference is that the configuration results in 
industrial control are used in real-time monitoring. The interpretation engine of industrial configuration tools should be run in real time according to the results of industrial configuration. On the face of it, the operating procedure of an industrial configuration tool is to perform a specific task. Industrial configuration software has the characteristics of real-time multi task, open interface, flexible operation and reliable operation. One of the most prominent features is the real-time multi task. It can complete the data acquisition at the same time on a computer, data processing, data display, can realize man-machine conversation, real-time data storage, historical data query, real-time multi task communication etc.. Industrial configuration software is the user of automation engineering design; industrial configuration software allows users to generate suitable for their own needs in the application system without the need to modify the software program source code.

\section{Monitoring System Design of Multiple Elevators Based on IoT and Industrial Configuration}

Design of Data Collecting Module. The module of elevator information management, user management, elevator maintenance records management and maintenance of various elevator related basic information management, and for property management, maintenance personnel and monitoring unit in charge. The competent authorities of the government can also carry out daily and monthly statistics on the maintenance of the elevator according to the area and the unit; the statistics are extended and the elevator is not maintained. Maintenance unit has the right to set up the elevator data management fault management and maintenance management functions. The use of network maintenance units can always check the elevator management, such as the elevator lift list, to maintain the elevator and elevator inspection list; on the other hand, it can also modify, supplement and update on the management of elevator related information, select settings management related constraints. In the software design, browse query is the main function. The query can be in accordance with the time range, elevator brand, maintenance unit, administrative area, maintenance personnel and elevator name query. A good man-machine interface is designed to update the data and information in order to meet the functional requirements of the system. According to the collected location, we call signal and control instruction to realize automatic orientation, smooth to cut a car, car, remote reverse cutting automatic switch door function; at the same time to lower frequency converter, gating device and signal display device, control the elevator down to open or close the door and display the current location of the elevator ladder. Lift the top speed and acceleration and deceleration time of elevator running through the elevator inverter set, enable and speed according to the configuration software to the direction of the enable signal to make inverter to lift acceleration, uniform speed, deceleration, leveling parking control, display power frequency or elevator running speed.

Design of Data Supervising Module. The monitoring information module is used to monitor the running state and fault condition of the elevator in real time. In the elevator safety monitoring center server, maintenance units and units in charge of security, can loop, loop lock remote dynamic monitoring the working state of the elevator, on the downstream and opening and closing conditions and other related safety parameters. In the monitoring information module, the fault message alarm function is set up. When the elevator is in trouble, the monitoring information module will inform the staff on the spot for the first time. If the relevant personnel within the set time range is not processed, the system will alarm information upgrade, automatically send the fault information to a higher level of the relevant responsible person in charge of the unit. In the group control system, the response of each elevator to the call signal outside the hall is assigned by the system. Outside the hall call signal is not sent directly to the passengers call the elevator controller, but the first to control scheduling module, scheduling module according to the current state information of the ladder, take certain deployment strategy, through the analysis of the algorithm which elevator to respond to the call signal will make the elevator system to get the optimal performance of the hall the call signal is assigned to the elevator controller. Therefore, the system has to control the elevator controller, in response to a command signal of an outbound elevator hall. In order to improve the transportation efficiency and reduce the waiting time of passengers, the elevator group scheduling algorithm based on minimum 
waiting time is adopted. The minimum waiting time scheduling principle is based on the new generation of call and calculates the elevator response time. We select the shortest response time to answer the call.

Design of Data Mining Module. Elevator safety monitoring data analysis system includes two parts, one is the analysis of the basic data in the monitoring terminal, and the two is the analysis of complex data in the server. The monitoring terminal through the analysis of the basic data of the monitoring data, the formation of the running state of the elevator data information, and the elevator running state parameters and standard parameters comparison, analyzing the current elevator operation status and fault conditions, when comparing the results beyond the standard parameter values when the fault information in the form of short message promptly sent to the relevant management personnel. The data analysis server mainly completed in a period of time of elevator status data, prediction feature extraction, and principal component analysis and trend, which will help data processing and data mining technology, to the aim of early warning of elevator fault information to achieve. If the phenomenon of overweight, the indicator light is overweight, the door is kept open and write abnormal state table, only when the overweight problem solved, the elevator will run normally. If the door open or close, there will be opening and closing fault, if the long time does not touch the lift up and down movement of upper and lower level sensor system will consider the fault of elevator traction machine, elevator stop running and malfunction indicator lamp lit, at the same time the configuration will send the fault characters for traction the monitor screen, the monitor screen will receive the signal to open the abnormal status report. Monitoring system through the data analysis and processing module, the data obtained will be analyzed and compared with the standard parameters of the elevator. When the result is beyond the range of the parameter value, the fault information is sent to the monitoring center and the maintenance unit respectively, and the duty officer is prompted in the form of language.

\section{Function Realization of Monitoring System of Multiple Elevators}

Problems Can Be Alarmed Timely. Elevator things through the agreement with the elevator controller communication, can accurately obtain the elevator information for the accident to provide an active alarm channel. Can be the first time to learn the elevator accident information and notify the relevant personnel through the system messages. Lift annual inspection by a special equipment inspection department and Technical Supervision Bureau, the elevator inspection qualified inspection report issued by qualified, in order to make the passengers understand the safety of the elevator, the elevator car is usually in a prominent position with a certificate in elevator inspection. The inspection department of the Technical Supervision Bureau expressly stipulates that the elevator needs to have the qualified company to carry on the maintenance, and provides the maintenance company to have to maintain two times every month. It is difficult to research the implementation of maintenance company safety problems. It is difficult to define the responsibilities, and even a few maintenance company, undocumented workers also forged certificates to deceive users.

Roots Can Be Traced Accurately. Elevator networking can also help business users to quickly locate the whole staircase elevator, at the same time the names of these address access to these units, the use of the elevator maintenance unit, installation units, property companies and other related units and contact, know the elevator inspection, elevator maintenance and elevator operation. To establish a real-time monitoring system database as a whole system of data processing, the core of the organization and management of the historical data of real time process, process for archiving, query historical data, accident analysis, experience learning. The force control of real-time database is a data object to index data, a point by a number of parameters, system parameters for the unit to store all kinds of information, which is equivalent to relational database keyword parameters equivalent fields. In the system, the speed of each detection point, the position of the floor, the upper and lower states are needed to be saved in the database. Because the monitoring system directly monitor and control the field goal, which requires data display and intuitive and eye-catching, can vary with the state of the object being monitored, so as to realize the real-time change of computer screen to reflect the 
dynamic operation process. Display interface mainly displays the real-time operation of the elevator, which is the state of open and close and up and down.

Experiences Can Be Shared Voluntarily. Elevator things can be set up knowledge sharing columns, dedicated to the accumulation of elevator maintenance experience and a variety of elevator knowledge. Users can share their experience and knowledge into knowledge sharing, you can also ask questions online, ask for answers. Other users can give answers to these questions at any time, and the answers will be automatically logged into the knowledge sharing section. The debugging information of the elevator, the fault information of the non-accident and the parameter information of the parts are all the private information of the whole enterprise. Private information can be transferred to the whole ladder through the Internet of things. Things themselves do not do any processing, only as an information channel. Elevator SMS alarm function can be set in the system has been added to the fault message alarm settings, when the elevator failure will be the first time after the notice to the person in charge, if within the time stipulated in the relevant personnel are not handled, the system will automatically be upgraded to send alarm by relevant competent and responsible person. Maintenance history inquiries can view the elevator maintenance history, to be well documented. The fault record query can view the fault record. If the record is more, you can use conditional search to narrow the scope of the record.

\section{Conclusion}

The elevator monitoring management system based on the Internet of Things and industrial configuration technology is used to realize the monitoring of the running state of multiple elevators and the coordination management of the elevator management department. All monitoring management work is carried out in the scene of personnel absence. It will bring great convenience to the elevator management, which has important social and economic benefits.

\section{Acknowledgement}

In this paper, the research was sponsored by the Education Department Foundation of Hubei Province of China under Grant No. B2015362.

\section{References}

[1] Chen Jiayan, Hong Tao, Liu Ganghai, Wang Guoqiang, Zhou Juan, Research of elevator safety monitoring system based on internet of things technology [J]. Journal of China Universit y of Metrology, 2013, 24(3): 231-236.

[2] Li Guangping, Deng Rukui, Fan Yi, Li Suling. Design of Elevator Experimental Model Based on PLC and Configuration Software [J]. Industrial Control Computer, 2016, 29(12): 133-134+143.

[3] Huang Qinlu, Yang Wei, Xiao Gan. The application of internet of things technology in the city's elevator monitoring and alarming management system [J]. Manufacturing Automation, 2013, 35(1):30-33.

[4] Liu Weixing. Discussion on the Design of Double Six Elevator Simulation System Based on Configuration Software [J]. Forestry Science and Technology Information, 2013, 45(3): 58-59. 